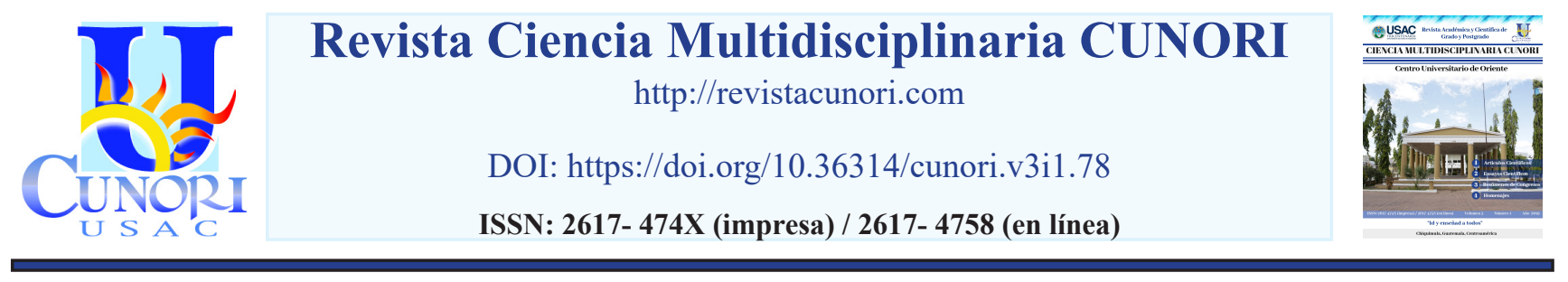

Como citar el artículo

Navarro, N., Mazariegos, E., Arriola, C., Aldana, A., Velásquez, G., (2019). Estado nutricional preoperatorio. Revista Ciencia Multidisciplinaria CUNORI, 3(1), 43-50. DOI: https://doi.org/10.36314/cunori.v3i1.78

\title{
Estado nutricional preoperatorio
}

\author{
Preoperative nutritional status
}

Kimberly Navarro*, Edvin Mazariegos, Carlos Arriola, Astrid Aldana, Gilberto Velásquez

Centro Universitario de Oriente (CUNORI), Universidad de San Carlos de Guatemala

Recibido: 30 de julio de 2018 / Revisión: 10 de septiembre de 2018 / Aceptado: 07 de enero de 2019

Disponible en internet el 30 de agosto de 2019

\author{
*Autor para correspondencia. \\ Resumen \\ Correo electrónico: kiminm_5@hotmail.com
}

\begin{abstract}
T a prueba de valoración global subjetiva (VGS) es una herramienta de evaluación que utiliza el método clínico de valoración _de riesgo nutricional a través de la historia clínica y la exploración física para conocer el estado nutricional del individuo. Se basa en datos subjetivos relacionados con: cambio de peso, cambio en ingesta dietética, presencia de síntomas gastrointestinales y capacidad funcional. Este estudio permitió determinar el estado nutricional preoperatorio en 50 pacientes de ambos sexos en edades de 20 a 70 años, que ingresaron para cirugía electiva al servicio de cirugía de adultos del Hospital Nacional de Chiquimula durante los meses de agosto de 2017 a abril de 2018. Resultados y discusión: La prueba muestra que de los 50 pacientes estudiados el $62 \%$ están bien nutridos, $32 \%$ en riesgo de desnutrición y $6 \%$ desnutridos, en el sexo masculino predominó el riesgo de desnutrición con un 55\%, el rango de edad que presentó mayor riesgo de desnutrición y desnutrición establecida fue 61 a 70 años con el $16 \%$ y $4 \%$ respectivamente, se determinó que de la población estudiada, 21 pacientes presentaron una pérdida de peso entre el 5 y $10 \%$ y 3 presentaron una pérdida mayor al 10\%, es decir una pérdida severa que indica un estado de desnutrición. El tipo de cambio en la ingesta alimenticia fue dieta sólida subóptima con el $44 \%$, el síntoma gastrointestinal más frecuente referido fue náusea con el $40 \%$, la alteración en la capacidad funcional fue 44\% y el signo clínico de desnutrición más frecuente fue pérdida de grasa con el 32\%. La prueba de valoración global subjetiva demuestra que 16 pacientes presentaron riesgo de desnutrición y 3 se encontraban desnutridos.
\end{abstract}

Palabras clave: prueba de valoración global subjetiva, estado nutricional, cirugía electiva

\begin{abstract}
$\mathrm{T}$ he subjective global assessment test (VGS) is an evaluation tool that uses the clinical method of nutritional risk assessment through the clinical history and physical examination to know the nutritional status of the individual. It is based on subjective data related to: weight change, change in dietary intake, presence of gastrointestinal symptoms and functional capacity. Materials and methods: This study determined the preoperative nutritional status in 50 patients of both sexes aged 20 to 70 years, who were admitted for elective surgery to the adult surgery service of the National Hospital of Chiquimula during the months of August of 2017 to April. of 2018. Results and discussion: The test shows that of the 50 patients studied $62 \%$ are well nourished, $32 \%$ at risk of malnutrition and $6 \%$ malnourished, in the male sex the risk of malnutrition was $55 \%$, the range of age that presented the highest risk of malnutrition and malnutrition was 61 to 70 years with $16 \%$ and $4 \%$ respectively, it was determined that of the studied population, 21 patients presented a weight loss between 5 and $10 \%$ and 3 presented a loss greater than $10 \%$, that is, a severe loss that indicates a state of malnutrition. The type of change in dietary intake was suboptimal solid diet with $44 \%$, the most frequent gastrointestinal symptom referred was nausea with $40 \%$, the alteration in functional capacity was $44 \%$ and the clinical sign of malnutrition more frequent was loss of fat with $32 \%$. The subjective global assessment test shows that 16 patients were at risk of malnutrition and 3 were malnourished.
\end{abstract}


Keywords: subjective global assessment test, nutritional status, elective surgery

\section{Introducción}

El estado nutricional es la situación en la que se encuentra una persona en relación con la ingesta y adaptaciones fisiológicas que tienen lugar tras el ingreso de nutrientes. Este depende de una variedad de factores como: comercialización, condiciones geográficas, ingresos, disponibilidad de alimentos, acceso, hábitos, creencias alimentarias, educación, cultura, utilización biológica de alimentos, enfermedad, higiene, saneamiento ambiental, estilos de vida y actividad física (Hott, 2014). La UNICEF define el estado nutricional como el estado de crecimiento, nivel de micronutrientes de un individuo o condición del organismo que resulta de la relación entre las necesidades nutricionales individuales y la ingestión, absorción y utilización de los nutrientes contenidos en los alimentos. La manera adecuada de determinar y comprobar si efectivamente se están cumpliendo éstas necesidades nutricionales es la evaluación, utilizando pruebas de cribaje como la prueba de valoración global subjetiva, la cual consiste en un método clínico de valoración de riesgo nutricional a través de la historia clínica y la exploración física (FAO, 2017).

La malnutrición hospitalaria fue descrita por Butterworth en 1974 en su artículo "El esqueleto en el closet", llamando la atención de la comunidad médica. Existe amplia documentación sobre la relación entre malnutrición y mayor morbilidad, mortalidad de los pacientes hospitalizados, así como del mayor tiempo de hospitalización, niveles más elevados de reingreso y aumento de los costos. Se suma a esta argumentación la valoración de los costos del soporte nutricional; como consecuencia de ello los estudios costo-beneficio exigen objetivos clínicos estrictos tales como reducción en las tasas de mortalidad, la incidencia de complicaciones y la duración de la estadía hospitalaria (Estévez, 2011).

El presente trabajo hace una descripción con fundamento teórico y de carácter clínico del estado nutricional preoperatorio en pacientes que fueron sometidos a cirugía electiva, en donde las estadísticas a nivel mundial demuestran que el riesgo de desnutrición en pacientes hospitalizados oscila entre $30 \mathrm{y}$ el 55\%, aumentando estas cifras hasta un 70\% al momento del alta hospitalaria, demostrando que la prevalencia de riesgo nutricional es alta ya sean éstos pacientes clínicos o quirúrgicos (Bordon, 2015).

El estado de deterioro nutricional es causa de un riesgo incrementado de hasta 20 veces para padecer complicaciones en comparación con los pacientes con adecuado estado nutricional. La incidencia de complicaciones va desde el $9 \%$ en pacientes con desnutrición moderada, hasta el $42 \%$ con desnutrición severa (Pérez, 2016). Por tal motivo es importante concientizar acerca de la importancia de una evaluación nutricional temprana a pacientes que se encuentren vulnerables y así disminuir los factores que puedan influir en el deterioro de su salud durante un procedimiento quirúrgico.

\section{Material y métodos}

El estudio es de tipo descriptivo transversal en pacientes de ambos sexos entre las edades de 20 a 70 años, que fueron sometidos a cirugía electiva ingresados en el departamento de cirugía de adultos del Hospital Nacional de Chiquimula. Para alcanzar dicho objetivo se utilizó la prueba de valoración global subjetiva la cual utiliza el método clínico de valoración de riesgo nutricional a través de la historia 
clínica y la exploración física, basándose fundamentalmente en la anamnesis nutricional en donde se obtienen datos relacionados con el cambio de peso en los últimos 6 meses, cambios en la ingesta dietética, presencia de síntomas gastrointestinales y capacidad funcional. Como resultado se obtiene una clasificación de los pacientes en bien nutridos, riesgo de desnutrición y desnutrición franca.

\section{Resultados y discusión}

De una población 50 pacientes se logró determinar que el 32\% presentó riesgo de desnutrición y $6 \%$ desnutrición, en el sexo masculino predominó el riesgo con un 55\%, el rango de edad que presentó mayor riesgo de desnutrición y desnutrición fue 61 a 70 años con el 16\% y 4\% respectivamente, se determinó que de la población estudiada, 21 pacientes presentaron una pérdida de peso entre el 5 y $10 \%$ y 3 presentaron una pérdida mayor al 10\%, es decir una pérdida severa que indica un estado de desnutrición. El tipo de cambio en la ingesta alimenticia fue dieta sólida subóptima con un 44\%, el síntoma gastrointestinal más frecuente referido fue náusea con el 40\%, la alteración en la capacidad funcional fue de 44\% y el signo clínico de desnutrición más frecuente fue pérdida de grasa con el 32\%. Demostrando de esta manera que los pacientes si presentan un riesgo de desnutrición y desnutrición a su ingreso.

\section{Clasificación del estado nutricional según VGS de los pacientes}

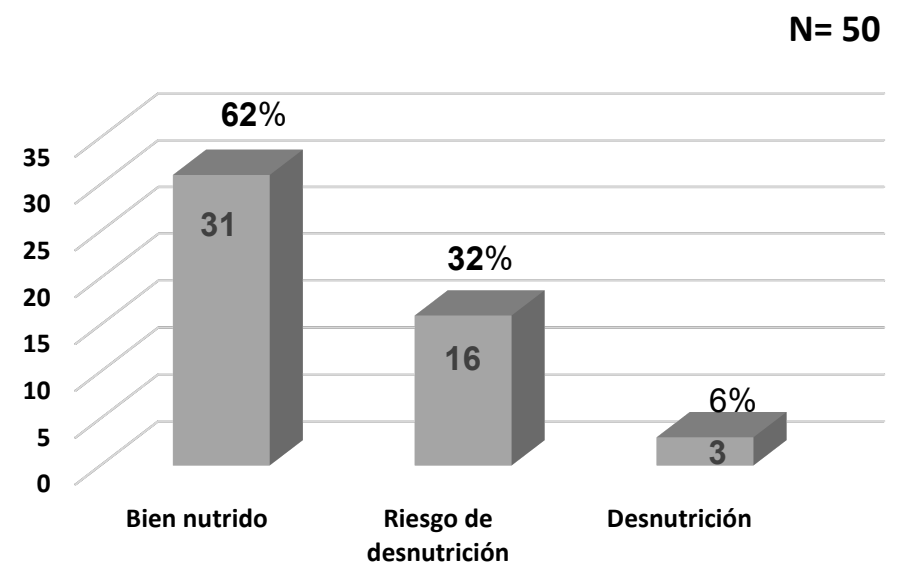

Figura 1. Clasificación del estado nutricional según VGS de los pacientes que fueron sometidos a cirugía electiva ingresados en el departamento de cirugía de adultos del Hospital Nacional de Chiquimula durante los meses de noviembre de 2017 a febrero de 2018.

Según la investigación realizada se encontró que 31 pacientes estaban bien nutridos, 16 presentaban riesgo de desnutrición y 3 desnutriciones, una situación de deterioro nutricional desemboca cuando las demandas orgánicas de nutrientes no se satisfacen, la desnutrición se puede deber a múltiples causas y una de ellas es la ingesta reducida de nutrientes. La falta de una ingesta adecuada de nutrientes se asocia con un deterioro significativo en relación al estado nutricional, esto sugiere que poder adecuar la ingesta de nutrientes puede ser un determinante importante de los resultados clínicos. Por ello la importancia de una evaluación precisa con el fin de identificar pacientes que se encuentren más vulnerables. 
Distribución del estado nutricional según género de los pacientes

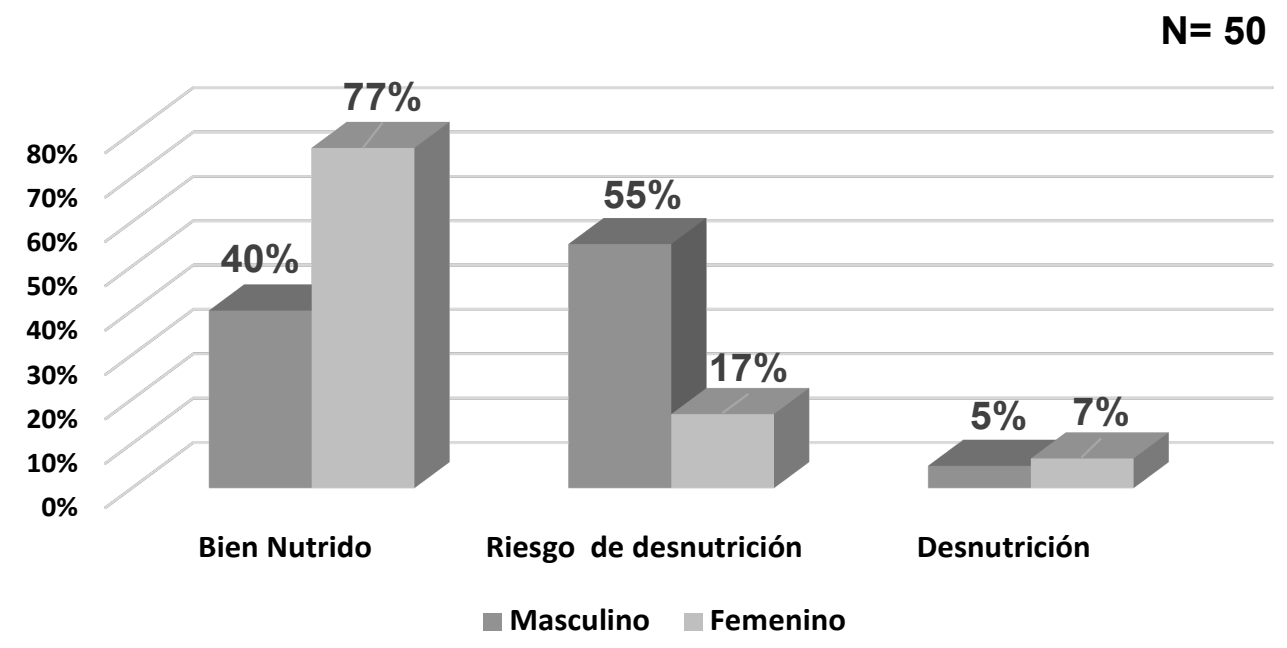

Figura 2. Distribución del estado nutricional según género de los pacientes que fueron sometidos a cirugía electiva ingresados en el departamento de cirugía de adultos del Hospital Nacional de Chiquimula durante los meses de noviembre de 2017 a febrero de 2018.

De la población en estudio destaca el riesgo total de desnutrición en el sexo masculino con un 55\% y $17 \%$ en el sexo femenino; debido a que las mujeres tienden a perder peso de forma más lenta y menos constante porque el metabolismo hormonal sufre cambios periódicos y tienden a retener mucho más líquido; por ello disponen de más grasa en el organismo y están predispuestas biológicamente a almacenarlas para la etapa reproductiva; los hombres por el contrario tienen un metabolismo más rápido y no pasan por estos procesos, por lo cual la tendencia a mayor riesgo se encuentra en ellos.

\section{Distribución del estado nutricional según edad de los pacientes}

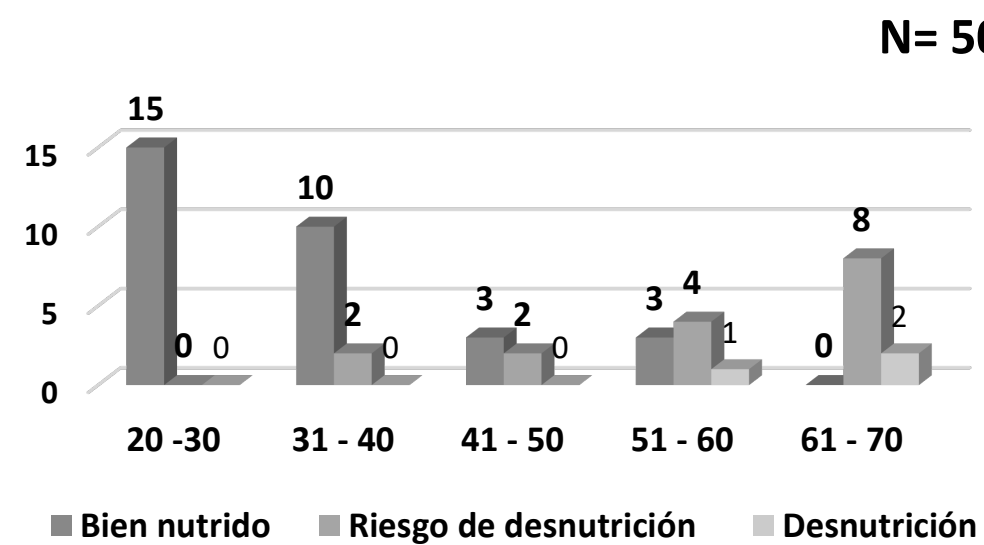

Figura 3. Distribución del estado nutricional según edad de los pacientes que fueron sometidos a cirugía electiva ingresados en el departamento de cirugía de adultos del Hospital Nacional de Chiquimula durante los meses de noviembre de 2017 a febrero de 2018. 
En relación a la edad de los pacientes estudiados; 8 de ellos se encuentran en riesgo de desnutrición y 2 en desnutrición en el rango de 61 a 70 años. El deterioro nutricional se presenta en este rango de edad ya que la población asociada a esto son los adultos mayores de 65 años. A medida que la edad avanza surgen cambios fisiológicos en la composición corporal, la masa magra y contenido mineral óseo. Existe un aumento de depósitos de grasas y modificación en la distribución de ésta, aumentando el tejido adiposo visceral mientras que disminuye el tejido graso subcutáneo. Como consecuencia de estos cambios, el gasto energético disminuye. De igual forma los niveles de actividad disminuyen originando un gasto energético total menor a medida que se envejece. Esta disminución del gasto energético total se asocia a una menor ingesta calórica; es decir que con la edad no se modifica únicamente la composición corporal sino también la adaptación metabólica a los cambios en la ingesta presentándose una mayor dificultad para alcanzar de nuevo el peso adecuado.

\section{Porcentaje de pérdida de peso de los pacientes}

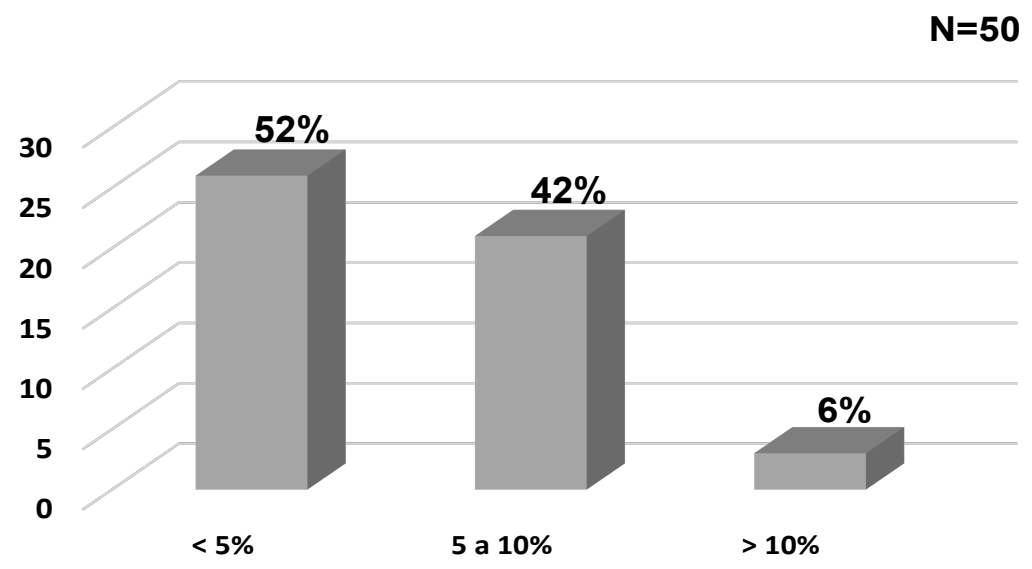

Figura 4. Porcentaje de pérdida de peso de los pacientes que fueron sometidos a cirugía electiva ingresados en el departamento de cirugía de adultos del Hospital Nacional de Chiquimula durante los meses de noviembre de 2017 a febrero de 2018.

En cuanto a la pérdida de peso, 26 pacientes tuvieron $<5 \%$ de porcentaje de pérdida, 21 entre el 5 a $10 \%$ y 3 perdieron más del 10\%; es decir, que los pacientes que pierden menos o igual al 5 y $10 \%$ en 6 meses están relacionados con una pérdida significativa que indica riesgo de desnutrición, en comparación con los pacientes que pierden más del 10\%; en los cuales la pérdida es severa e indica desnutrición. Se ha demostrado que pérdidas mayores al 10\%, van acompañadas de afectaciones fisiológicas y alteraciones en la ingesta; siendo esto claramente indicativo de mayor incidencia de complicaciones postoperatorias y aumento de estancia hospitalaria. 
Distribución del tipo de cambio en la ingesta dietética de los pacientes

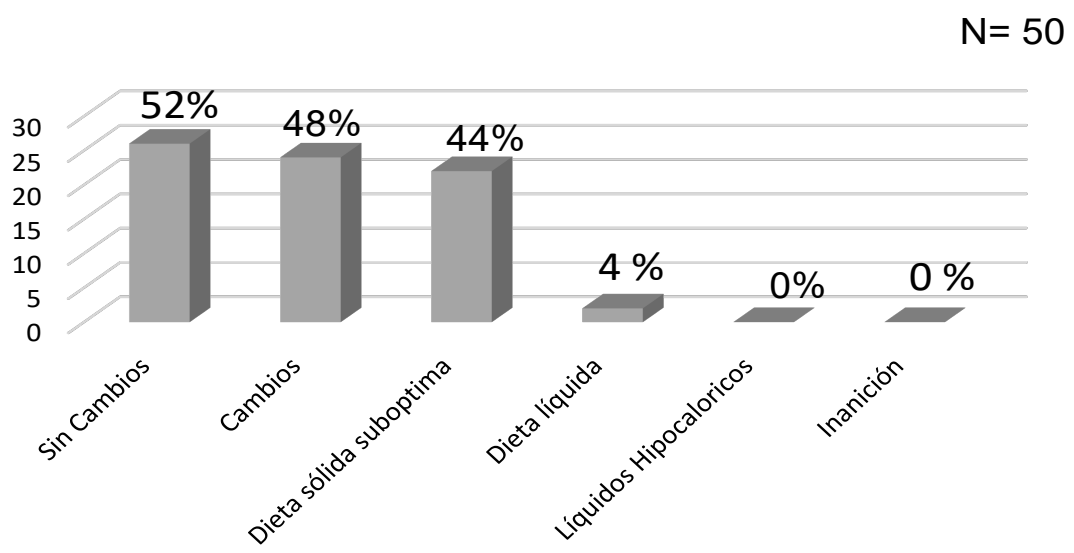

Figura 5. Distribución del tipo de cambio en la ingesta dietética de los pacientes que fueron sometidos a cirugía electiva ingresados en el departamento de cirugía de adultos del Hospital Nacional de Chiquimula durante los meses de noviembre de 2017 a febrero de 2018.

De los 50 pacientes en estudio 24 si presentaron cambios en la ingesta dietética; de acuerdo al hecho de que la elección más importante que la población puede hacer para influir a medio y a largo plazo en su salud es el consumo de una dieta óptima; el primer condicionante para que la dieta sea nutricionalmente equilibrada es que estén presentes en ella la energía y todos los nutrientes necesarios, en cantidades adecuadas y suficientes para cubrir las necesidades nutricionales de cada individuo y evitar deficiencias. Sin embargo en este estudio los pacientes modificaron estos hábitos consumiendo una dieta subóptima y líquida; esto debido a que los trastornos digestivos pudieron tener influencia, ya que dentro de los síntomas gastrointestinales que refirieron los pacientes en el momento del interrogatorio nutricional en orden de frecuencia de mayor a menor fueron: náusea con el $40 \%$, vómito $26 \%$, dolor $24 \%$ y anorexia con el 20\%; siendo todo en conjunto la consecuencia de estos cambios.

\section{Capacidad funcional de los pacientes que fueron sometidos a cirugía electiva}

$\mathbf{N}=\mathbf{5 0}$

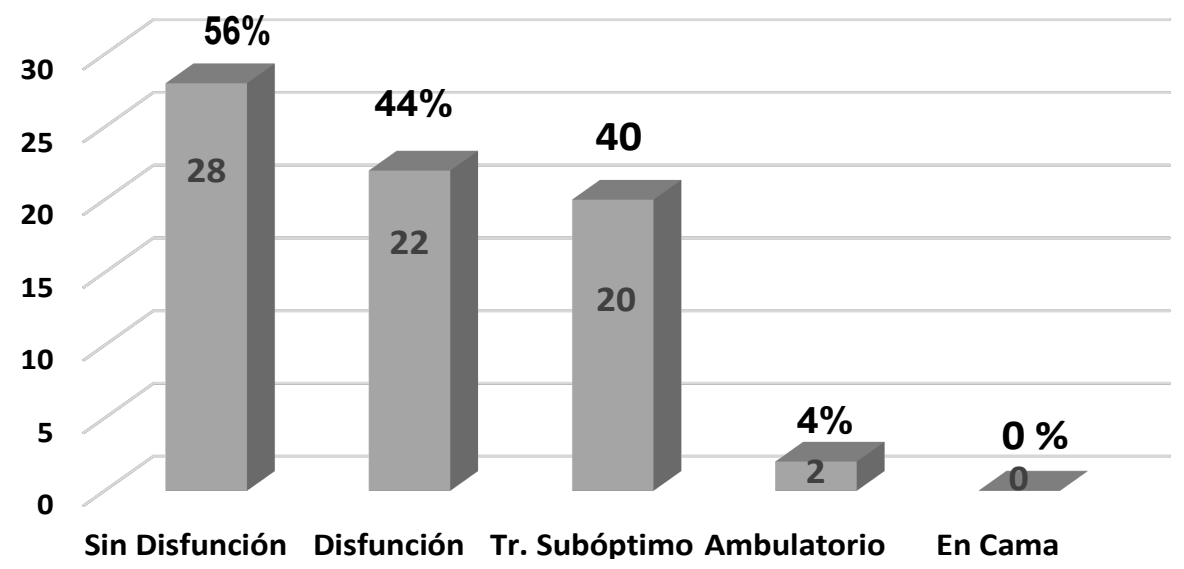

Figura 6. Capacidad funcional de los pacientes que fueron sometidos a cirugía electiva ingresados en el departamento de cirugía de adultos del Hospital Nacional de Chiquimula durante los meses de noviembre de 2017 a febrero de 2018. 
Se demuestra que el trabajo subóptimo con el $40 \%$ y ambulatorio representado el $4 \%$ fueron los tipos de disfunción que presentaron los pacientes en estudio; esto debido a que hubo una pérdida significativa de peso en los pacientes que se encontraban en riesgo de desnutrición, siendo este un fenómeno que suele acarrear consecuencias como perdida de la fuerza muscular; deteriorando el estado de salud y dando origen a repercusiones en su capacidad funcional.

\section{Referencias bibliográficas}

Bordon, C. 2015. Evaluación del estado nutricional y del riesgo nutricional en pacientes quirúrgicos ¿por qué, cuándo y cómo realizarlas? (en línea). Archivos Latinoamericanos de Nutrición 65(sup. 1). Consultado 15 ago. 2017. Disponible en https://www.alanrevista.org/ediciones/2015/suplemento-1/ art-228/\#

Estévez, R. 2011. Desnutrición intrahospitalaria ELAN, Ecuador: Hospital Carlos Andrade Marin, Quito, provincia de Pichincha, 2011 (en línea). Tesis M.Sc. Riobamba, Ecuador, Escuela Superior Politécnica de Chimborazo, EPEC. 172 p. Consultado 28 ago. 2017. Disponible en http://dspace.espoch. edu.ec/ bitstream/123456789/3974/1/20T00497.pdf

FAO (Organización de las Naciones Unidas para la Alimentación y la Agricultura, Italia). 2017. Evaluación nutricional (en línea, sitio web). Roma, Italia. Consultado 10 sep. 2017. Disponible en http:// www.fao.org/nutrition/evaluacion-nutricional/es

Hott Novoa, M. 2014. Texto - guía de evaluación del estado nutricional: material de apoyo para la asignatura de evaluación del estado nutricional de los alumnos de la carrera de nutrición y dietética (en línea). Arica, Chile, Universidad de Tarapacá, FACSAL. p. 1-17. Consultado 14 ago. 2017. Disponible en http://sb.uta.cl/libros/ GUIAevaluacionnutricional2014.pdf

Pérez-Flores, JM; Chavez-Tostado, M; Larios-del-Torro, YE; García-Rentería, J; Rendón-Félix, J; Salazar-Parra, M; Irusteta-Jiménez, L; Michel-Espinoza LR, Márquez-Valdez, AR; Cuesta-Márquez, L; Álvarez-Villaseñor, A; Fuentes-Orozco, C; Gonzáles-Ojeda, CA. 2016. Evaluación del estado nutricional al ingreso hospitalario y su asociación con la morbilidad y mortalidad en pacientes mexicanos ( en línea). Revista Nutrición Hospitalaria 33(4):872-878. Consultado 19 ago. 2017. https:// doi.org/10.20960/nh.386

\section{Sobre la autora}

\section{Kimberly Lucrecia Navarro Méndez}

Médico y Cirujano, egresada del Centro Universitario de Oriente CUNORI de la Universidad de San Carlos de Guatemala en el año 2018. Realizó la investigación que lleva como título "Estado nutricional preoperatorio". 


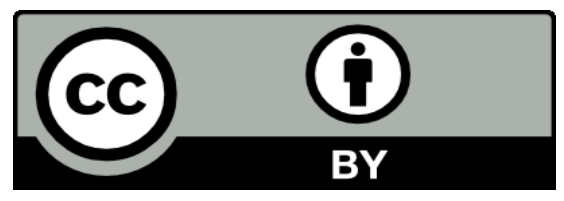

Este texto está protegido por una licencia CreativeCommons 4.0.

Usted es libre para compartir, copiar y redistribuir el material en cualquier medio o formato y adaptar el documento, remezclar, transformar y crear a partir del material para cualquier propósito, incluso comercialmente, siempre que cumpla la condición de atribución: usted debe reconocer el crédito de una obra de manera adecuada, proporcionar un enlace a la licencia, e indicar si se han realizado cambios. Puede hacerlo en cualquier forma razonable, pero no de forma tal que sugiera que tiene el apoyo del licenciante o lo recibe por el uso que hace. 